\title{
INTERACTIONS CULTURALISTES ET BIOPOLITIQUES EN TEMPS DE PANDÉMIE : STRATÉGIES D'ACTION ET INTÉRÊTS DES UTILISATEURS DE YOUTUBE DE MARS À JUIN 2020
}

\section{ARTICLE ORIGINAL}

DENDASCK, Carla Viana ${ }^{1}$, OLIVEIRA, Euzébio de ${ }^{2}$

DENDASCK, Carla Viana. OLIVEIRA, Euzébio de. Interactions culturalistes et biopolitiques en temps de pandémie : stratégies d'action et intérêts des utilisateurs de Youtube de mars à juin 2020. Revista Científica Multidisciplinar Núcleo do Conhecimento. An 05, Ed. 08, vol. 16, p. 38 et 47. août 2020. ISSN: 24480959, Lien d'accès: https://www.nucleodoconhecimento.com.br/communicationfr/culturalistes-et-biopolitiques, DOI: 10.32749/nucleodoconhecimento.com.br/communication-fr/culturalistes-etbiopolitiques

\section{RÉSUMÉ}

Cet article, réalisé dans le cadre de l'accomplissement des disciplines du Programme doctoral en communication et sémiotique organisé à I'Université Pontificale Catholique de São Paulo, vise à réfléchir sur les interactions culturelles et biopolitiques adoptées en pleine épidémie de Covid-19 au Brésil entre mars et juin 2020. Pour cela, nous avons utilisé l'analyse des 50 chaînes les plus consultées sur Youtube au cours des 4 derniers mois, en examinant si et comment les utilisateurs manifestent leurs comportements et leurs intérêts à travers leurs recherches respectives dans ces médias. Grâce à ces résultats, une brève analyse a été

\footnotetext{
${ }^{1}$ Théologien, docteur en psychanalyse clinique. Il travaille depuis 15 ans avec la Méthodologie Scientifique (Méthode de Recherche) dans I'Orientation de Production Scientifique pour les étudiants en Master et Doctorat. Spécialiste en études de marché et recherche en santé.

${ }^{2}$ Biologiste, docteur en maladies tropiques, professeur et chercheur du cours d'éducation physique, Université fédérale du Pará (UFPA).
}

RC: 89284

Disponible en: https://www.nucleodoconhecimento.com.br/communication- 
réalisée sur l'efficacité des politiques mises en œuvre et leurs conséquences possibles.

Mots-clés : Pandémie, interactions culturelles et biopolitiques, Youtube.

\section{INTRODUCTION}

Depuis que les médias techniques ont commencé à exprimer les manifestations des cultures, des intérêts et des tendances collectives, certes, la société n'avait pas rencontré de crise causée par l'annonce de l'état de pandémie, réalisée par l'Organisation mondiale de la santé en mars 2020, résultant de la nouvelle COVID19 , avec recommandation d'isolement social, qui a remodelé les routines des villes , conduisant des centaines de milliers de personnes à utiliser leurs appareils technologiques (smartphones, ordinateurs portables, ordinateurs) comme moyen de communication prioritaire, que ce soit dans la routine de travail, les études, la socialisation, entre autres.

Malgré cet état qualifié de chaotique, on ne peut nier que ce contexte a créé un environnement propice à des réflexions profondes, et a ouvert les portes à un champ fertile d'études dans tous les domaines, en particulier dans le domaine de la communication. II est entendu que même l'existence d'une extension de lieux et d'espaces, ceux qui ont été initialement délimités et discutés comme des lieux fixes et des espaces ouverts, publics et privés, prennent des dimensions complexes. Bien que nous s'accordions avec Belting (2009, p.81) que les espaces ont les caractéristiques d'être organisés de manière hétérogène et discontinue, dans le cyberespace créé par le scénario pandémique, qui transcendait auparavant ces dimensions, ils ont alors commencé à travailler dans une sphère de déplacement et de compréhension du temps et de l'espace, puisque leurs limites brisent les barrières physiques et commencent à se manifester dans une ambiguïté, parfois contradictoire, contribuant parfois à de nouvelles façons de vivre, penser, organiser, travailler, célébrer, ressentir, relier, entre autres.

RC: 89284

Disponible en: https://www.nucleodoconhecimento.com.br/communication- 
Lors des cours du doctorat en Communication Sémiotique à l'Université Pontificale Catholique de São Paulo, en ce premier semestre 2020, le champ de réflexion et d'études ne s'est pas éloigné de ce contexte présenté. D'abord en raison de la nécessité d'une adaptation rapide des routines, en particulier dans les changements d'environnements en face à face à l'environnement en ligne, à la fois par les étudiants qui ont connu des adaptations de nouvelles routines d'étude, ainsi que par les enseignants qui ont dû apprendre un nouveau système de communication, un système qui obéit à certains processus et langues qui leur sont propres. II y a aussi le scénario de l'enseignement de la classe elle-même, où les enseignants et les élèves ont dû s'adapter à de nouveaux formats d'interactions pendant les cours. Ce contexte a certainement causé le sentiment initial d'éloignement.

Plus tard, on ne peut pas mentionner que tous les textes, bien qu'ils aient été précédemment choisis dans cet état pandémique, ont servi à approfondir les réflexions contemporaines, presque comme s'ils avaient été prévus. Peut-être parce que les domaines d'études et les thèmes abordés par la communication ont ce pouvoir intemporel, ou, peut-être, à cause de la maîtrise des enseignants dans l'appropriation des thèmes et l'apport de réflexions à nos jours. Plus que jamais, il a été compris que sans moyens techniques, il n'est pas possible de parler dans la communication en tant que science, ainsi, il a été démontré que la technologie est fondamentale pour la survie de la communication en tant que domaine de connaissance.

Ces contributions ont fait les collectes mensuelles des 50 chaînes les plus consultées sur Youtube qui sont menées dans le cadre des investigations de la thèse finale, ainsi que l'analyse de leurs stratégies de communication étant résignées dans la mesure où les cours et les textes travaillés démontraient de nouveaux modes de manifestations et possibilités de compréhension des intérêts des utilisateurs.

Rappelons que l'intérêt pour la plateforme Youtube est notamment dû au fait que, sur cette plateforme, l'utilisateur "choisit» ce qu'il veut regarder, contrairement à

RC: 89284

Disponible en: https://www.nucleodoconhecimento.com.br/communication- 
d'autres médias, tels que la télévision qui effectue une programmation basée sur d'autres stratégies. Sur la plateforme youtube, la rapidité de communication, la démocratisation des producteurs de contenus dans différents formats, la pluralité des publics, les formes d'interactions et les actions à émerger dans la mesure « à ce qui est choisi » permettent à cette plateforme d'exprimer les intérêts d'une partie importante de la société contemporaine, soit par niche, soit dans le collectif.

Ainsi, ce matériel a été organisé en deux moments: Le premier faisant une brève allusion à la pandémie, comment les politiques ont été mises en œuvre et la création d'une communication de panique et de peur par les principaux médias au Brésil. Dans le deuxième moment, nous avons montré comment cela se reflétait dans les recherches sur les réseaux sociaux, en particulier sur youtube. Enfin, les concluants font quelques réflexions sur certains aspects qui impliquent les intérêts et les stratégies qui sont absorbés par les utilisateurs de ce réseau.

\section{BRÈVES CONSIDÉRATIONS SUR LA PANDÉMIE / LA COMMUNICATION ET LES BIOPOLITIQUES DE CONTRÔLE}

Le coronavirus est une espèce de virus mutant connue des chercheurs depuis les années 1970. Cependant, la variante connue sous le nom de COVID-19 ou SARSCoV-2 a été découverte au second semestre 2019 à Wuhan, en Chine. Ce qui a conduit l'Organisation mondiale de la santé à considérer ce virus comme une pandémie, c'est son pouvoir élevé de propagation et sa capacité à évoluer vers une pneumonie aiguë, considérée comme à haut risque de morbidité et de mortalité pour certains groupes d'individus (SILVA et. al. 2020).

L'ignorance de son origine, de ses variables d'évolution et de ses conséquences a conduit les autorités du monde entier à adopter un discours homogène et à prendre des mesures restrictives sévères, conduisant la population à une situation de panique. La société, des leaders aux petits groupes, a rapidement mis en évidence l'incapacité émotionnelle à faire face à la peur, avec impuissance à travers la vie, en

RC: 89284

Disponible en: https://www.nucleodoconhecimento.com.br/communication- 
prenant des mesures qu'Agamben (2020) a qualifiées de frénétique et irrationnelle, sans études multidisciplinaires comme conséquences, adoptant un discours "pour la vie " qui a fini par conduire à des centaines d'autres problèmes socioéconomiques, ainsi que la santé publique, comme la dépression, l'anxiété, le suicide, l'obésité, entre autres.

Selon Agamben (2009, p. 50), «plus les appareils se répandent et répandent leur pouvoir dans tous les domaines de la vie, plus le gouvernement est confronté à un élément incompréhensible [...] ». Dans le processus pandémique, les sujets ont été à la merci des biopolitiques gouvernementales, des dispositifs de contrôle ont été mis en place et ont rapidement vu l'installation de diverses formes d'autres crises, telles que la crise économique, la crise entre les pouvoirs.

Dans la société contemporaine, de tels dispositifs, bien qu'ils agissent de manière percutante et directe, sont également capables de faire passer les développements au-delà de la compréhension des tendances, qui finissent par être responsables des nouvelles formes de biopolitique. Sur la COVID-19, Agamben (2020) a souligné que la peur, la peur d'infecter et d'infecter d'autres personnes ont conduit les biopolitiques de la restriction de la liberté à être approuvées, même sans de nombreuses discussions sur ses conséquences, par une grande partie de la population mondiale. Les autorités mondiales et les médias se sont réunis pour répandre le sentiment de panique, affirmant la tendance croissante de l'état d'exception qui, dans ce cas, a permis à une sorte de " militarisation » de faire ce que cela signifie avec toute une société de la part de ceux qui ont le pouvoir.

Pendant un instant, il a oublié la " multiplicité des drames sociaux » pour ne regarder qu'un seul problème, seulement une focalisation, et en son nom, accepter une dictature voilée, et d'une certaine manière, soutenue par divers publics, en utilisant des techniques biopolitiques.

RC: 89284

Disponible en: https://www.nucleodoconhecimento.com.br/communication- 
Selon Lazzarato (2006, p.81): «Les techniques que la biopolitique est adressée à la vie, sont dirigées vers l'être vivant comme appartenant à l'espèce humaine. Ils visent à réguler la vie affectée par la maladie, le chômage, la vieillesse, la mort.

Le gros problème est que cette action de contrôle a conduit à une crise sociale de la pensée, des positions polarisées qui ont réduit le problème réel de la pandémie à des positions et des conditions personnelles, empêchant les gens d'agir ou de penser collectivement, cependant, en utilisant des termes tels que « au nom de la vie ", les groupes ont été divisés, et les dirigeants des hommes d'État ont utilisé ces scénarios pour élaborer des politiques et des biopolitiques de contrôle qui agissaient sur le principe et le droit à la liberté , en utilisant divers groupes d'intérêt, en plus de s'approprier ces conditions afin que les politiques publiques d'urgence permettent l'absence d'offres, ce qui conduirait à un scénario pour une autre crise, la « corruption ».

Entre mars et mai, la télévision ouverte a créé un climat de panique et suspendu la programmation normale pour mettre l'accent de manière exhaustive sur la pandémie. Ce qui devrait être de l'information est également devenu un scénario politique et a créé une sorte de vide entre les actions et les conduites nécessaires et celles effectuées, mettant l'accent sur le moulage des corps, mais maintenant de manière ambiguë. La société de contrôle a connu son dédain, c'est-à-dire que les progrès de l'information et de la technologie se poursuivent, mais que faire de cette information pour produire des biopolitiques positives en temps de crise s'il y a une crise existentielle de la peur? Cette réponse ne semble toujours pas avoir été intégrée, même par la société, ni par ses dirigeants. Cependant, quelques indices sur ces développements peuvent être trouvés à travers les manifestations d'intérêts selon les recherches sur les réseaux sociaux, comme nous le démontrerons dans la séquence de cette étude.

RC: 89284

Disponible en: https://www.nucleodoconhecimento.com.br/communication- 


\section{YOUTUBE COMME FORME DE MANIFESTATION D'INTÉRÊTS}

Comme mentionné précédemment, pour recueillir les données de la thèse de doctorat, sont étudiées les 50 canaux les plus consultés de Youtube et les stratégies utilisées par ces canaux dans la création de contenu pour atteindre leurs utilisateurs respectifs. Après mars 2020, la période qui a commencé la quarantaine, nous avons observé la création de tendances qui n'existaient pas auparavant, telles que la prévalence de la vie des chanteurs, l'intérêt lié à la COVID-19 au cours des deux premiers mois et le fort désintérêt après le mois de mai. D'autre part, il a également été démontré l'utilisation de cette plate-forme dans le secteur de l'éducation par certains États, ce qui a conduit à un accès accru pour le public des enfants et des jeunes. Au cours de cette période, Youtube a revu ses politiques internes et a décidé de ne pas monétiser les chaînes destinées à ces publics, et il y a eu alors une baisse de la production de contenu destiné à ce groupe d'âge. Vous trouverez ci-dessous le tableau des 50 vidéos des chaînes youtube les plus consultées.

Tableau 1 - 50 vidéos les plus consultées

\begin{tabular}{|c|c|c|c|c|c|c|c|c|}
\hline Catégorie & Mars & & Avril & & Mai & & Juin & \\
\hline & $\begin{array}{l}\text { La } \\
\text { quantit } \\
\text { é }\end{array}$ & $\%$ & $\begin{array}{l}\text { La } \\
\text { quantit } \\
\text { é }\end{array}$ & $\%$ & $\begin{array}{l}\text { La } \\
\text { quantit } \\
\text { é }\end{array}$ & $\%$ & $\begin{array}{l}\text { La } \\
\text { quantit } \\
\text { é }\end{array}$ & $\%$ \\
\hline COVID & 6 & $12 \%$ & 4 & $8 \%$ & 2 & $4 \%$ & 0 & $0 \%$ \\
\hline Vivre & 0 & 0 & 1 & $2 \%$ & 15 & $30 \%$ & 22 & $\begin{array}{l}44 \\
\%\end{array}$ \\
\hline Musique & 6 & $12 \%$ & 8 & $16 \%$ & 4 & $8 \%$ & 0 & $0 \%$ \\
\hline BBB & 3 & $6 \%$ & 3 & $6 \%$ & 0 & $0 \%$ & 0 & $0 \%$ \\
\hline $\begin{array}{l}\text { Commentaire } \\
\text { du film }\end{array}$ & 3 & $6 \%$ & 2 & $4 \%$ & 0 & $0 \%$ & 0 & $0 \%$ \\
\hline "Vlogueiros" & 6 & $12 \%$ & 3 & $6 \%$ & 3 & $6 \%$ & 5 & 10 \\
\hline
\end{tabular}

RC: 89284

Disponible en: https://www.nucleodoconhecimento.com.br/communication- 


\begin{tabular}{|c|c|c|c|c|c|c|c|c|}
\hline $\begin{array}{l}\text { Démonstration } \\
\text { de routines }\end{array}$ & & & & & & & & $\%$ \\
\hline $\begin{array}{l}\text { Culture de } \\
\text { l'Asie de l'Est }\end{array}$ & 1 & $2 \%$ & 1 & $2 \%$ & 1 & $2 \%$ & 4 & $8 \%$ \\
\hline $\begin{array}{l}\text { Exercices } \\
\text { physiques }\end{array}$ & 3 & $6 \%$ & 1 & $2 \%$ & 2 & $4 \%$ & 0 & $0 \%$ \\
\hline $\begin{array}{l}\text { Commentaires } \\
\text { sur la beauté } \\
\text { esthétique }\end{array}$ & 1 & $2 \%$ & 0 & $0 \%$ & 1 & $2 \%$ & 1 & $2 \%$ \\
\hline alimentation & 6 & $12 \%$ & 4 & $8 \%$ & 4 & $8 \%$ & 1 & $2 \%$ \\
\hline $\begin{array}{l}\text { Evangile/ } \\
\text { Religieux }\end{array}$ & 2 & $4 \%$ & 2 & $4 \%$ & 3 & $6 \%$ & 1 & $2 \%$ \\
\hline $\begin{array}{l}\text { Chaînes } \\
\text { d'acteurs } \\
\text { célèbres } \\
\text { télévision }\end{array}$ & 4 & $8 \%$ & 3 & $6 \%$ & 1 & $2 \%$ & 0 & $0 \%$ \\
\hline Sport & 2 & $4 \%$ & 1 & $2 \%$ & 1 & $2 \%$ & 2 & $4 \%$ \\
\hline Voiture & 1 & $2 \%$ & 1 & $2 \%$ & 0 & $0 \%$ & 1 & $2 \%$ \\
\hline $\begin{array}{l}\text { Ordinateurs/ } \\
\text { Ordinateurs }\end{array}$ & 0 & 0 & 3 & $6 \%$ & 0 & $0 \%$ & 1 & $2 \%$ \\
\hline Jeu & 3 & $6 \%$ & 3 & $6 \%$ & 2 & $4 \%$ & 5 & $\begin{array}{l}10 \\
\%\end{array}$ \\
\hline $\begin{array}{l}\text { Comédie/Humo } \\
\text { ur }\end{array}$ & 1 & $2 \%$ & 1 & $2 \%$ & 1 & $2 \%$ & 4 & $8 \%$ \\
\hline $\begin{array}{l}\text { Science et } \\
\text { éducation }\end{array}$ & 2 & $4 \%$ & 4 & $8 \%$ & 3 & $6 \%$ & 2 & $4 \%$ \\
\hline $\begin{array}{l}\text { Maison, } \\
\text { Décoration et } \\
\text { Jardin }\end{array}$ & 0 & 0 & 0 & $0 \%$ & 0 & $0 \%$ & 1 & $2 \%$ \\
\hline
\end{tabular}

RC: 89284

Disponible en: https://www.nucleodoconhecimento.com.br/communication$\underline{\text { fr/culturalistes-et-biopolitiques }}$ 


\begin{tabular}{|l|l|l|l|l|l|l|l|l|}
\hline Horoscope & 0 & $0 \%$ & 2 & $4 \%$ & 0 & $0 \%$ & 0 & $0 \%$ \\
\hline $\begin{array}{l}\text { Entreprises et } \\
\text { entrepreneuriat }\end{array}$ & 0 & $0 \%$ & 3 & $6 \%$ & 3 & $6 \%$ & 0 & $0 \%$ \\
\hline Commérage & 0 & $0 \%$ & 0 & $0 \%$ & 3 & $6 \%$ & 0 & $0 \%$ \\
\hline Total & 50 & $\begin{array}{l}100 \\
\%\end{array}$ & 50 & $\begin{array}{l}100 \\
\%\end{array}$ & 50 & $\begin{array}{l}100 \\
\%\end{array}$ & 50 & 100 \\
\hline
\end{tabular}

Source : Propre

Dans le tableau ci-dessus, il est possible de percevoir certains développements du contexte pandémique à travers les intérêts des utilisateurs. En mars, lorsque la politique d'isolement a été créée et qu'il y avait encore beaucoup d'ignorance du virus, les canaux qui ont abordé le covid-19 se sont démarqués parmi les plus consultés de la chaîne (12\%) des 50 canaux les plus consultés. Même au cours des deux premiers mois (mars et avril), l'une des chaînes les plus consultées avait un contexte de terreur pandémique et de prédiction apocalyptique du virus. Cependant, au fil des mois, l'intérêt des utilisateurs régresse jusqu'à ce que, en juin, l'intérêt pour le thème n'atteigne pas les 50 vidéos quotidiennes les plus regardées.

D'autre part, la création de lives et leur représentativité dans l'accès et l'intérêt des utilisateurs, atteignant le pourcentage de $44 \%$ (parmi les 50 vidéos) des vidéos les plus consultées, démontrent que ces utilisateurs sont avides de divertissement, en particulier les événements qui apportent l'image / la mémoire des événements sociaux.

Il est également à noter qu'au début de la pandémie, les intérêts étaient plus pulvérisés, il y avait un grand intérêt pour la pratique de l'exercice physique à la maison, commentant les films et le divertissement. Cependant, au fil des mois, ces intérêts diminuaient. De même, les chaînes du célèbre, qui ont commencé à avoir une fréquentation de contenu et, par conséquent, ont promu un format d'accessibilité

RC: 89284

Disponible en: https://www.nucleodoconhecimento.com.br/communication- 
pertinente, avec le passage des mois semble avoir diminué leur intérêt pour la production de ce type de contenu.

Déjà en avril, lorsque les premières traces de crise économique ont commencé à être évidentes, en particulier par la fermeture des métiers, les canaux de l'entrepreneuriat et des affaires avaient une forte demande qui, peu de temps après, a cessé de susciter l'intérêt.

II existe certains types de chaînes qui maintiennent leur niveau de demande et de production de contenu, présentant un certain pourcentage de constance dans les mois, parmi lesquels: cuisine, jeux, éducation, sport et les vlogs qui démontrent la vie quotidienne de certains youtubeurs.

\section{CONSIDÉRATIONS FINALES}

L'étude de la communication en tant que science est en mesure de fournir des subventions pour divers domaines de la connaissance, ainsi que pour la société. Ses signes et représentations à travers sa dynamique fournissent également une base pour comprendre les développements possibles, car il était possible d'observer à travers les données collectées sur Youtube.

Les intérêts des utilisateurs dans les mois de la pandémie montrent qu'au départ, il y avait une plus grande peur et une recherche de compréhension par cette population d'utilisateurs. Cependant, il présente, au fil des mois, un désintérêt collectif, probablement dû aux dimensions politiques et aux actions polarisées que ce thème a prises au Brésil. II semble également que la population ne soit pas enthousiasmée par la routine de la maison. L'intérêt pour la vie, et la proportion telle qu'elle apparaît dans la liste des faits saillants majeurs, indiquent également une population à la recherche d'une sorte d'abstraction par le divertissement et l'anxiété dans la socialisation de ce qui peut provoquer un effet de troupeau des réunions et des fêtes avant la période souhaitée, aggravant encore les effets de cette pandémie. Enfin,

RC: 89284

Disponible en: https://www.nucleodoconhecimento.com.br/communication- 
cette étude poursuivra ses collectes mensuelles respectives, fournissant encore plus de données pour les analyses futures avec un niveau de profondeur plus élevé.

\section{RÉFÉRENCES}

AGAMBEN, Giogio. O que é o contemporâneo? E outros ensaios. Ed. Argos, Chapecó, 2009.

AGAMBEN, Giorgio et. al. Sopa de Whuan. ASPO. 2020

BELTING, Hans. Antroplogia de la imagem. Katz Editores. Madrid, 2009.

LAZZARATO, Maurizio. As revoluções do capitalismo. Ed. Civilização brasileira . Rio de Janeiro, 2006

SILVA, Anderson Walter Costa, et al. Perfil epidemiológico e determinante social do COVID-19 em Macapá, Amapá, Amazônia, Brasil. Revista Científica Multidisciplinar Núcleo do Conhecimento. Ano 05, Ed. 04, Vol. 04, pp. 05-27. Abril de 2020. ISSN: 2448-0959, Link de acesso: https://www.nucleodoconhecimento.com.br/saude/covid19-em-macapa, DOI: 10.32749/nucleodoconhecimento.com.br/saude/covid-19-emmacapa

Soumis : Août 2020.

Approuvé : Août 2020.

RC: 89284

Disponible en: https://www.nucleodoconhecimento.com.br/communication- 